INTERNATIONAL JOURNAL OF SCIENTIFIC RESEARCH

EFFECTS OF SILVER DIAMINE FLUORIDE ON EROSION OF DECIDUOUS ENAMEL BY LIQUID MEDICAMENTS: AN IN VITRO SURFACE MORPHOLOGICAL STUDY

\title{
Dental Science
}

Dr. Parvathy Suresh*

\section{Dr. Sobha} Kuriakose

Dr. Joy Kurian

\section{Dr. Soumya Rajan}

\section{Dr. Reshmi Raghuvaran}

Post Graduate Student, Department of Pediatric Dentistry,Sri Sankara Dental College,Varkala, Kerala, India. ${ }^{*}$ Corresponding Author

Dean, Sri Sankara Dental College, Varkala, Kerala, India.

Professor and HOD, Department of Pediatric Dentistry, Sri Sankara Dental College, Varkala, Kerala, India.

Reader, Department of Pediatric Dentistry, Sri Sankara Dental College,Varkala, Kerala, India.

Senior Lecturer, Department of Pediatric Dentistry, Sri Sankara Dental College,Varkala, Kerala, India.

\section{ABSTRACT}

Aim: To assess the protective effects of Silver Diamine Fluoride (SDF) against pediatric liquid medicament - induced enamel demineralization of deciduous teeth. Materials and methods: Ninety deciduous incisors were equally divided into three groups of 30 teeth each: without SDF pretreatment (group 1), with SDF pretreatment (group 2) and control group in artificial saliva (group 3). Group 1 and 2 were again divided into 3 subgroups of 10 each, according to the immersion solutions: sodium valproate, salbutamol sulphate and multivitamin. Vickers microhardness was measured at the pre-immersion stage and at regular intervals(7,14,21,28 days). Qualitative analysis was done using Scanning Electron Microscope (SEM) and the values were tabulated for statistical analysis. Results and Discussion: Evaluating the results of hardness tests, all the three medicines investigated could produce evident decrease in Vickers microhardness of all the tested enamel surfaces. Statistically significant erosion occurred $(\mathrm{p}<0.05)$ at all tested days in the multivitamin group, while sodium valproate and salbutamol were able to cause significant change only at the end of the 28 day cycle. The application of $38 \%$ SDF on the teeth samples prior to the erosive cycles was able to significantly reduce enamel erosion i.e, the reduction in hardness caused by erosion was statistically lesser in the SDF pretreated group. SEM images after 28 days revealed that all the tested surfaces clearly exhibited structural loss, which was unlike those immersed in artificial saliva. Conclusion: The qualitative and quantitative analysis of enamel samples pretreated with silver diamine fluoride suggested desirable protective effects of SDF on enamel against drug-induced erosive changes.

\section{KEYWORDS}

pediatric liquid medicaments; dental erosion; silver diamine fluoride; Vickers microhardness; scanning electron microscope.

\section{INTRODUCTION}

Erosion is defined as a progressive loss of dental hard tissues by chemical dissolution without bacterial involvement. ${ }^{1}$ It is a multi factorial irreversible process that may be caused by intrinsic, extrinsic or idiopathic factors. ${ }^{2}$ Erosion is frequently documented in individuals consuming fruit juices and carbonated soft drinks, and also in those patients taking certain acidic medicines regularly and long term. ${ }^{3}$ Liquid medicines have a long history of use in pediatric age group because of their ease of administration, dosing flexibility and convenience of a single formulation over a wide age range. In children with chronic or recurrent health problems, the long term use of PLMs may be inevitable.

Conventional fluorides, whose beneficial effect against caries is well known, have been tested for the prevention or control of erosion. ${ }^{4}$ Erosion cannot be prevented with moderate fluoride concentrations such as commonly used in toothpastes. ${ }^{5}$ Promising results have been obtained with a high fluoride concentration application.

Silver diamine fluoride (SDF) is a colorless liquid containing silver particles and $38 \%(44,800 \mathrm{ppm})$ fluoride ions, at $\mathrm{pH} 10$. It is $25 \%$ silver, $8 \%$ ammonia, $5 \%$ fluoride, and $62 \%$ water. It has the unique ability to be a "silver - fluoride bullet", simultaneously halting and preventing demineralization.

In the background of limited studies demonstrating the effects of SDF on enamel erosion, the current study aimed at assessing the protective role of SDF against PLM - induced enamel demineralization of deciduous teeth.

\section{MATERIALSAND METHODS}

Ninety extracted/exfoliated non - carious deciduous incisors were selected and stored at room temperature till experimental period in $10 \%$ formalin solution. The teeth were hand scaled and cleaned with pumice - water slurry and were examined by transillumination under high magnification to discard those with cracks, fractures or structural abnormalities that could interfere with the results.
The medications used were suspensions of sodium valproate, salbutamol sulphate and multivitamin. These drugs were chosen because they are syrups that are commonly used in pediatric patients for prolonged periods. The control group medium chosen was artificial saliva.

After removing the roots from all samples, each crown was fixed with wax in the central orifice of an acrylic plate with only the enamel surface exposed. The test sites were demarcated by attaching a round piece of insulating tape of $2 \mathrm{~mm}$ diameter. Teeth were coated with two layers of cosmetic nail polish, except for the previously delimited area. Vickers microhardness was assessed on the uncoated enamel area using a microhardness tester on all the 90 specimens and their averages were considered as the initial surface microhardness values.

The 90 deciduous incisors were equally divided into three groups of 30 teeth each: without SDF pretreatment (group 1), with SDF pretreatment (group 2) and control group in artificial saliva (group 3). Group 1 and 2 were again divided into 3 subgroups of 10 each, according to the immersion solutions: sodium valproate (group 1a,2a), salbutamol sulphate (group 1b,2b), multivitamin (group1c,2c).

Immersion Cycles: The specimens were immersed with the exposed area up for 1 minute in $10 \mathrm{~mL}$ of the medication, under agitation twice daily with 12 - hour intervals between the immersion cycles, during 7 days (14 immersion cycles). After each immersion cycle, the specimens were washed with distilled water and maintained in $10 \mathrm{~mL}$ of artificial saliva at $37^{\circ} \mathrm{C}$, until the next immersion cycle. The microhardness was then measured after a week. This process was repeated for 4 weeks, totalizing 56 immersion cycles. The medicines were replaced before each immersion. The control specimens were kept in artificial saliva during the course of the experiment (28 days) with the solution refreshed daily.

Surface microhardness was tested at 7, 14, 21 and 28 days after the continuous and systematic repetition of the daily immersion cycles. 
Results were statistically analyzed using the independent $t$ - test with the help of SPSS Version 21 software. SEM analysis was performed at the $28^{\text {th }}$ day of the experiment in five teeth from each group to observe the deciduous enamel changes.

\section{RESULTS}

Statistical analysis of the hardness values revealed that all the three tested liquid medicaments were capable of causing erosive changes in the tested enamel samples (graph 1). Statistically significant erosion occurred at all tested days in the multivitamin group, while sodium valproate and salbutamol were able to cause significant change only at the end of the 28 day cycle.

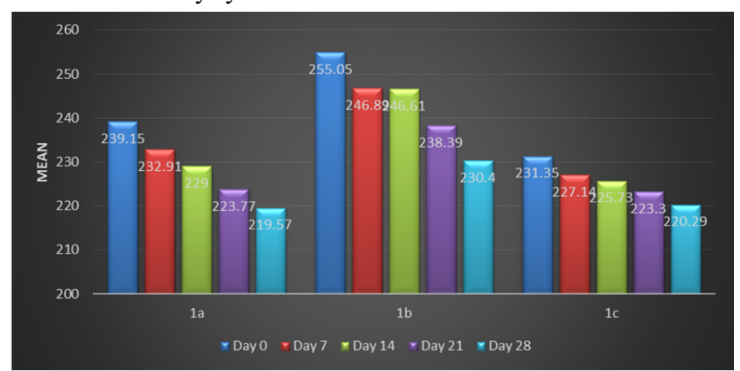

Graph 1: Histogram comparing the erosive potential of three commonly used pediatric liquid medicaments

The time $\times$ immersion media interaction demonstrated that in group 1 samples, a significant loss in surface microhardness was observed at all times. Meanwhile in group 2 (SDF pretreated), eventhough there were erosive changes, a statistically significant decrease was not obtained (graph 2).

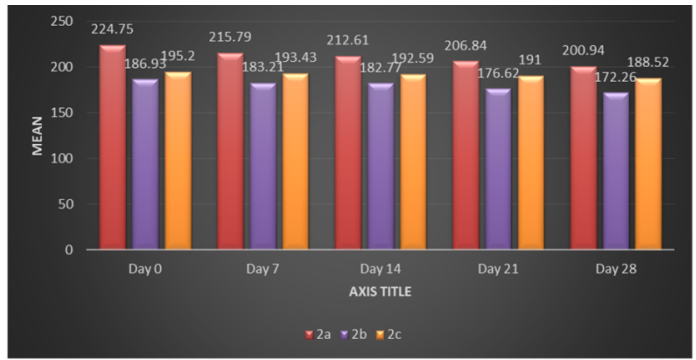

Graph 2: Histogram comparing the erosive potential of three commonly used pediatric liquid medicaments in the SDF pretreated samples

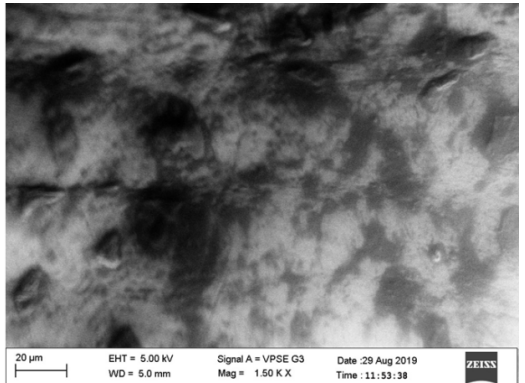

Figure 1: Group 1a: Crater formation seen in scanning electron microscope after exposure to sodium valproate at the end of the 28 - day cycle.

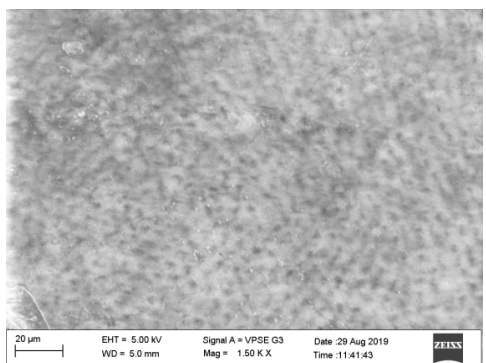

Figure 2: Group 2a: Etched prism pattern seen in scanning electron microscope after exposure to sodium valproate at the end of the 28 - day cycle.

At the end of the 28 - day cycle, SEM images of the non-SDF pretreated groups revealed crater formation (figure 1) after exposure to sodium valproate (1a) and salbutamol (1b), sporadic rod ends in multivitamin (1c) whereas etched prism patterns (figure 2) were observed in all SDF pretreated samples. In the control group, no surface changes were seen.

\section{DISCUSSION}

Despite dental caries being one of the most prevalent diseases of the oral cavity, tooth dissolution can also be caused by erosion, which in its severe form can be a predisposing factor for dental caries. This condition is of increasing concern as it causes irreparable damage to the dentition in subjects of all ages.

Studies by Linnett and Seow ${ }^{7}$ indicate that the prevalence of dental erosion has increased, especially among children and adolescents. All acids, whether from intrinsic(dietary acids and ones originated from the stomach ) or extrinsic sources, are capable of demineralizing tooth tissue and therefore of causing erosion. ${ }^{8}$ Extrinsic sources include acidic drinks, fruits and certain medications, especially those used for the treatment of chronic diseases.

The possible factors that contribute to the risk for dental erosion include acidic components, high frequency of ingestion (two or more times a day), bedtime consumption, high viscosity, and the collateral effect of a reduction in salivary flow.

The current study was designed to understand the erosive potential of select liquid medications and the protective effects, if any, of fluoride on enamel surfaces. The three syrups used in this study were selected because of their routine use in treating common pediatric diseases.

The experimental period (28 days) was chosen to simulate what would happen over a long treatment and it is highly possible that a longer treatment period might incur greater damages to the tooth structure than observed in this study. Based on the observation made by Amaechi BT ${ }^{10}$ that when a substance is ingested, a certain agitation occurs, which favors the substance's capacity to cause erosion, an agitation protocol was followed in the current study.

In the present study, an artificial saliva medium was used between the erosive immersion cycles to simulate in vivo conditions of the normal mouth and the normal remineralizing potential of fresh human saliva. ${ }^{11}$ In view of primary teeth being less mineralized and immature than permanent teeth and more prone to erosion ${ }^{12}$, the current study was conducted on deciduous teeth.

It is apparent that the protective effect of fluoride against erosive substance loss is due to the establishment of a $\mathrm{CaF}_{2}$ - like layer on the enamel surface. ${ }^{13}$ Silver diamine fluoride was selected as the material of choice in the current study based on the observations from many previous studies that erosion cannot be prevented with moderate fluoride concentrations but with high concentrations of fluoride such as professionally applied fluorides. ${ }^{14}$ Silver fluoride is the active agent in SDF when it is applied onto the tooth surface.

Studies by Rosenblatt et al. $2009{ }^{16}$ revealed that when applied to teeth, SDF penetrates enamel and dentin and 2 to 3 times more subsurface fluoride is stored in the tooth than with other fluoride solutions.

All the three medicines investigated could produce evident decrease in Vickers microhardness and observable changes in SEM. The results also suggested that the application of $38 \%$ SDF on the teeth samples prior to the erosive cycles was able to significantly reduce enamel erosion i.e, the reduction in hardness caused by erosion was statistically lesser in the SDF pretreated group.

\section{CONCLUSION}

The findings of the present study can be summarized as follows:

1. All the tested liquid medicaments viz. sodium valproate, salbutamol and multivitamin, caused erosive changes on the enamel surfaces, with significant reduction of enamel microhardness and structural alterations.

2. In the enamel samples pretreated with silver diamine fluoride, the quantitative analysis using Vickers microhardness and the qualitative analysis using scanning electron microscopy showed desirable protective effects against drug - induced erosive changes. 
In spite of the fact that SDF would provide a new preventive strategy against enamel erosion, for children having to consume long term medications, broader study sets are required to conclusively decide on alternative preventive protocols and risks of SDF application.

\section{Financial Support and Sponsorship: $\mathrm{Ni}$}

\section{Conflict of interest: $\mathrm{Nil}$}

\section{REFERENCES}

Scatena C, Galafassi D, Gomes-Silva JM, Borsatto MC, Serra MC. In Vitro Erosive Effect of Pediatric Medicines on Deciduous Tooth Enamel. Brazilian Dental Journal. 2014 Jan;25(1):22-7.

2. Mantonanaki M, Koletsi-Kounari H, Mamai-Homata E, Papaioannou W. Dental erosion prevalence and associated risk indicators among preschool children in Athens, Greece. Clinical oral investigations. 2012 Apr 25;17.

3. Nunn JH. Prevalence of dental erosion and the implications for oral health. Eur J Oral Sci. 1996 Apr;104(2 (Pt 2)):156-61.

4. Wiegand A, Attin T. Influence of fluoride on the prevention of erosive lesions--a review. Oral Health Prev Dent. 2003;1(4):245-53.

5. Larsen MJ, Richards A. Fluoride is unable to reduce dental erosion from soft drinks. Carse

6. Lagerweij MD, Buchalla W, Kohnke S, Becker K, Lennon AM, Attin T. Prevention of erosion and abrasion by a high fluoride concentration gel applied at high frequencies. Caries Res. 2006;40(2):148-53.

7. Linnett V, Seow WK. Dental erosion in children: a literature review. Pediatr Dent. 2001 Feb;23(1):37-43

8. Lussi A, Jaeggi T, Zero D. The role of diet in the aetiology of dental erosion. Caries Res. 2004;38 Suppl 1:34-44.

9. McDonald JL, Stookey GK. Laboratory studies concerning the effect of acid-containing beverages on enamel dissolution and experimental dental caries. J Dent Res. 1973 Apr;52(2):211-6.

10. Amaechi BT, Higham SM. In vitro remineralisation of eroded enamel lesions by saliva. J Dent. 2001 Jul;29(5):371-6.

11. Roberts IF, Roberts GJ. Relation between medicines sweetened with sucrose and dental disease. BrMed J. 1979 Jul 7;2(6181):14-6.

12. Johansson A-K, Omar R, Carlsson GE, Johansson A. Dental Erosion and Its Growing Importance in Clinical Practice: From Past to Present. International Journal of Dentistry. 2012;2012:1-17

13. Büyükyilmaz T, Øgaard B, Rølla G. The resistance of titanium tetrafluoride-treated human enamel to strong hydrochloric acid. European Journal of Oral Sciences. 1997; 105(5):473-7.

14. Ogard B, Seppä L, Rølla G. Professional topical fluoride applications--clinical efficacy and mechanism of action. Adv Dent Res. $1994 \mathrm{Jul} ; 8(2): 190-201$

15. Yamaga R, Nishino M, Yoshida S, Yokomizo I. Diammine silver fluoride and its clinical application. J Osaka Univ Dent Sch. 1972 Sep; 12:1-20

16. Rosenblatt A, Stamford TCM, Niederman R. Silver Diamine Fluoride: ACaries "SilverFluoride Bullet." Journal of Dental Research. 2009 Feb;88(2):116-25. 